SUPPLEMENTARY MATERIALS:

\title{
Enhanced structural and magnetic coupling in a mesocrystal-assisted nanocomposite
}

Yuanmin Zhu ${ }^{1)}$, Qian Zhan ${ }^{1)}$, Jan-Chi Yang ${ }^{2)}$, Yugandhar Bitla ${ }^{2)}$, Pingping Liu ${ }^{1)}$, Chen-I Li ${ }^{2)}$, Heng-Jui Liu ${ }^{2)}$, V. Suresh Kumar ${ }^{2)}$, Elke Arenholz ${ }^{3)}$, Qing He ${ }^{4)}$ and Ying-Hao $\mathrm{Chu}^{2,5)} *$

1) Department of Material Physics and Chemistry, University of Science and Technology Beijing, Beijing 100083, China; ${ }^{2)}$ Department of Materials Science and Engineering, National Chiao Tung University, Hsinchu 30010, Taiwan; ${ }^{3)}$ Advanced Light Source, Lawrence Berkeley National Laboratory, Berkeley, CA 94720, USA; ${ }^{4)}$ Department of Physics, Durham University, Durham DH1 3LE, United Kingdom; ${ }^{5)}$ Institute of Physics, Academia Sinica, Taipei 155, Taiwan

*Address corresponding to E-mail: Prof. Q.Zhan: qzhan@mater.ustb.edu.cn; Prof. Y.H.Chu: yhc@nctu.edu.tw 


\section{High-resolution reciprocal space maps:}

High-resolution reciprocal space maps (HRRSM) were carried out to probe the in-plane strain state in the both films shown in Figure. S1. The situation is similar to previous HRTEM analysis: we find fully relaxed strain states in the post-annealed sample while in the as-grown sample, the two constituent phases are strained due to the strong elastic interaction.
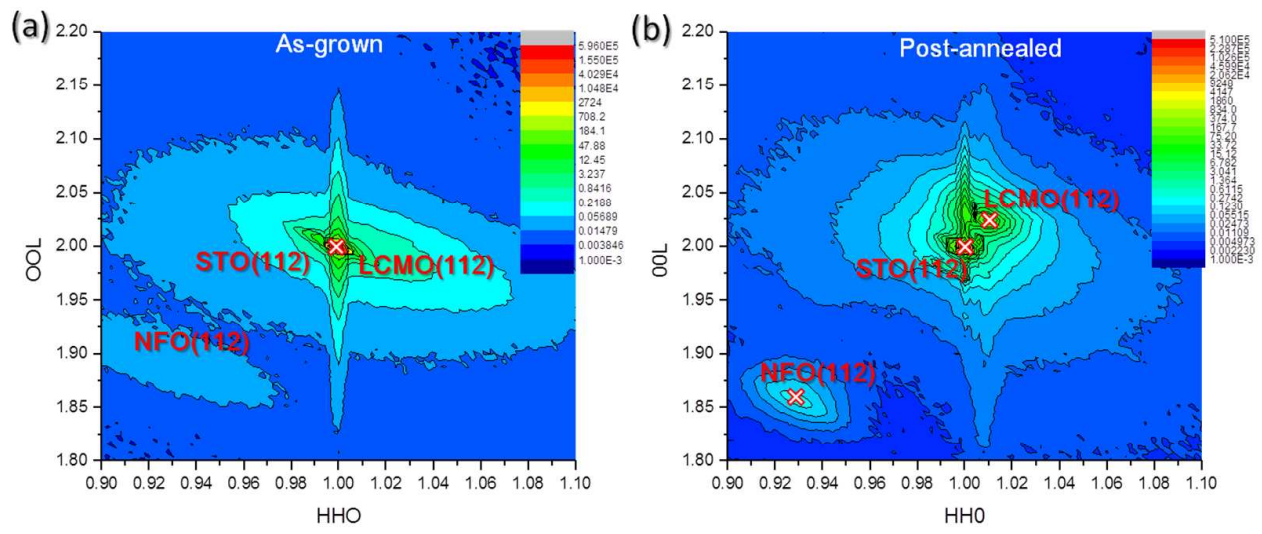

Figure S1 High-resolution reciprocal space maps of as-grown (a) and post-annealed (b) samples, respectively. The positions of the bulk value reflections for each phase were marked by cross in the maps.

\section{X-ray diffractions for the as-grown and post-annealed samples}




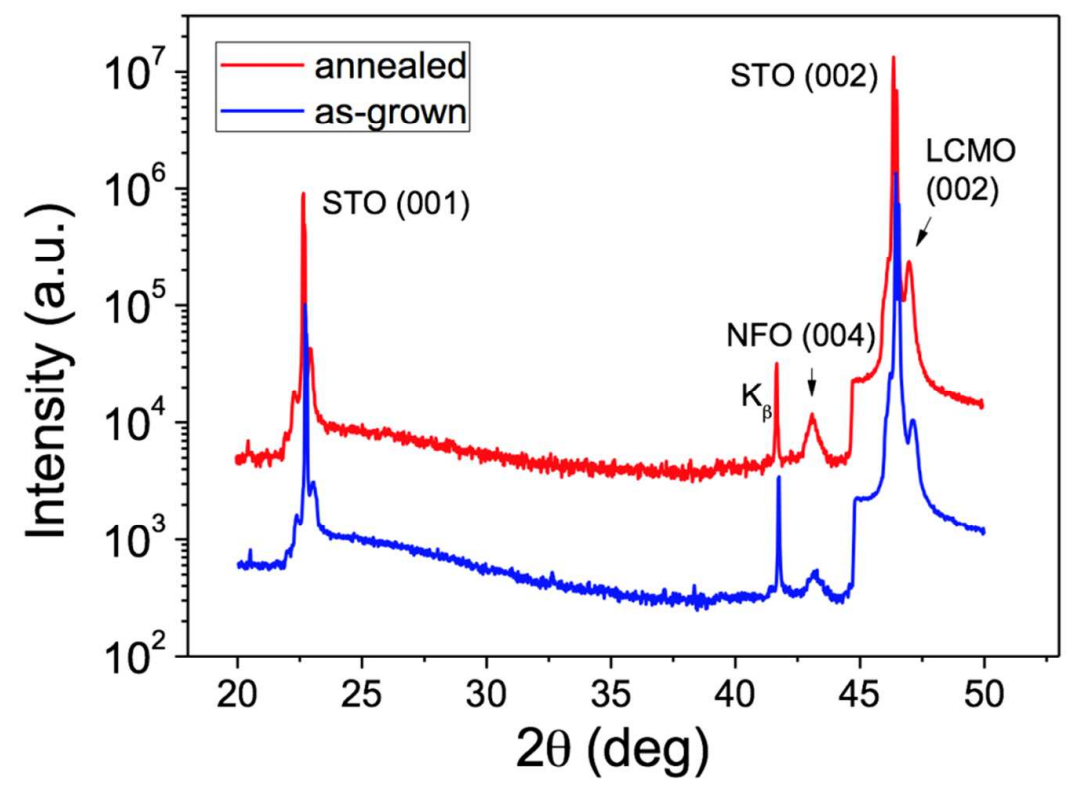

Figure S2 the X-ray diffractions for both the as-grown and post-annealed samples

3. quantitative analysis of the EDX spectrum in Fig.1e

Table S1 EDX quantification of the NFO and LCMO phases in the nanocomposite films (atomic\%).

\begin{tabular}{ccccccc}
\hline & $\mathrm{Ni}$ & $\mathrm{Fe}$ & $\mathrm{Mn}$ & $\mathrm{O}$ & $\mathrm{La}$ & $\mathrm{Ca}$ \\
\hline NFO & 9.15 & 15.41 & $\mathbf{8 . 2 8}$ & 67.13 & 0.00 & 0.00 \\
LCMO & 0.51 & $\mathbf{4 . 5 4}$ & 9.48 & 66.89 & 13.32 & 5.24 \\
\hline
\end{tabular}

\section{HRTEM interface analysis in the as-grown LCMO-NFO films}
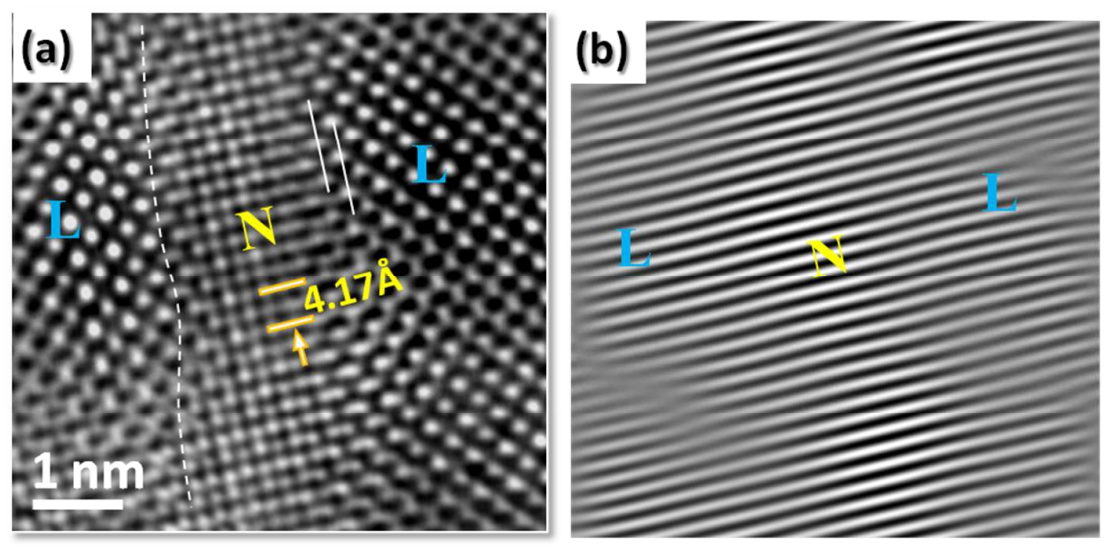
Figure S3 HRTEM analysis of LCMO-NFO heterointerface in the as-grown films. (a) the HRTEM image in Fig.3b. (b) the IFFT image of the LCMO(002)-NFO(004) interface. "L", "N" represent for LCMO, NFO phase, respectively.

\section{M-T curves for pure LCMO films}

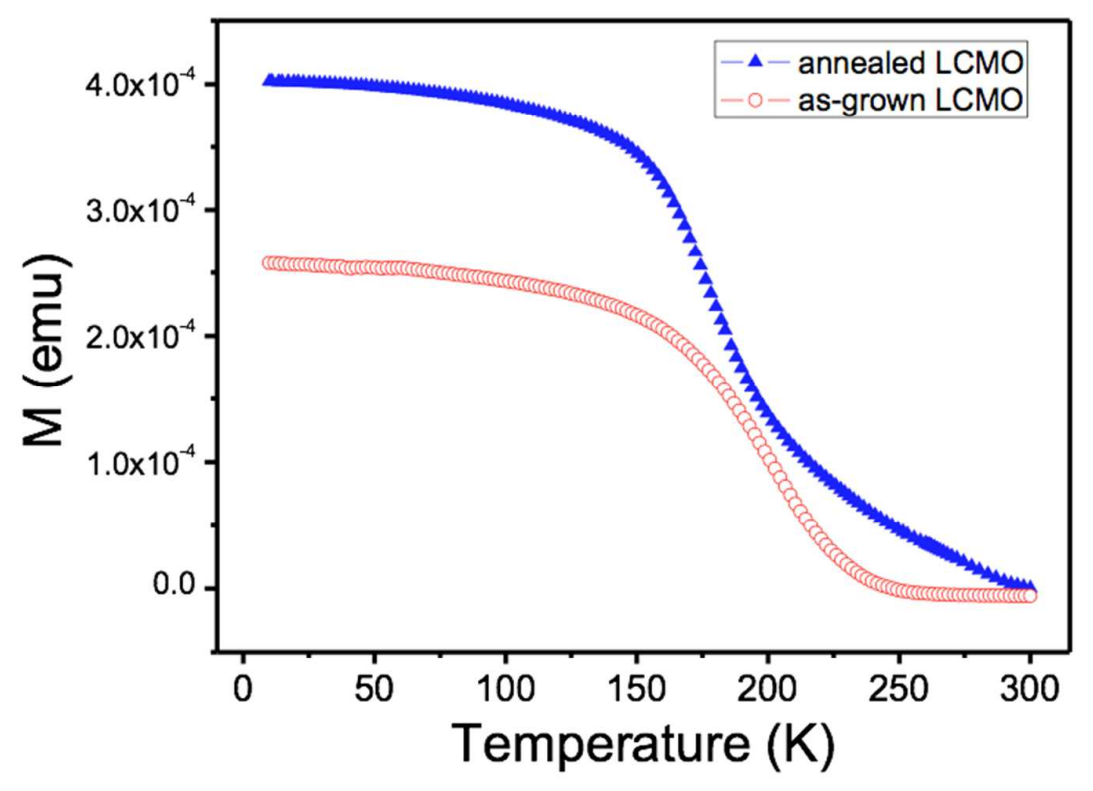

Figure S4 Temperature dependence of magnetization at $\mathrm{H}=1 \mathrm{kOe}$ for as-grown and post-annealed pure LCMO. 